\title{
Mise en valeur \\ par les grands travaux d'hydraulique des régions françaises déshéritées
}

\section{The recovery of waste land in France by large scale hydraulic methods}

\author{
Étude du Centre Français des Hautes Études Administratives \\ par MM. Abrial, Administrateur civil de l'Algérie; Auzelle, Urbaniste en \\ chef au M.R.L.; Bourrières, Ingénieur des Ponts et Chaussées à la France \\ d'Outre-Mer; Crucioni, Administrateur civil au Ministère de l'Agriculture; \\ Eynard, Ingénieur en chef du Génie Rural; Herbulot, Sous-Préfet; \\ Leroux, Administrateur de la France d'Outre-Mer; Yvon, Contrôleur civil \\ du Maroc.
}

\begin{abstract}
Sous-développement et grands projets (Métropole, Afrique du Nord, France d'Outre-Mer). Conduite des études. Eléments à étudier. Direction et coordination des études. - Réalisation du projet. Expropriation et remembrement. Echelonnement de tranaux. Financement. Exploitation des périmètres. Précédents en France et à l'étranger. Principaux modes d'exploitation envisagés. Choix du mode d'exploifation en fonction des dimensions du périmètre et des considérations sociales. - Conclusions.
\end{abstract}

\begin{abstract}
Large scale projects and under-development (in France, North Africa and French teritories overseas). - The line followed by studies. Points to be studied. Irirection and coordination of sludies.-Carying out the project. Requisitioning and redistribution of private property. Finance-Development of the areas under con'sideration. Precedents in France and in other lands. Main methods of development that are envisaged. Choice of method of development dependent upon size of area and social considerations.-Conclusions.
\end{abstract}

\section{SOUS-DEVELOPPEMENT ET GRANDS PROJETS}

\section{I. - Introduction}

Les régions de France sous-développées le sont généralement pour une raison physique, tenant à la géographie.

Le cas qui paraît le plus favorable c'est celui des marais que l'on peut assécher, des régions arides que l'on peut irriguer, des pays inactif's où l'on peut fabriquer de l'électricité à bon compte.

Ce qui confirme dans cette idée, c'est que l'on doit admettre que les générations qui se sont succédé sur les terroirs de France ont depuis toujours recherché les meilleures mises en valeur et qu'on n'a de chances d'amener un changement radical que là où l'on peut apporter des ćléments absolument nouveaux : c'est le cas des mises en valeur régionales par de grands travaux d'hydraulique.

Nous sommes dans le cas où la technique esl sûre d'apporter une amélioration durable, définitive même, à des régions entières.

C'est par les grands travaux que nous saisissons le plus directement la nature. C'est là qu'on doit obtenir les résultats les plus rapides, les plus spectaculaires. C'est aussi en ce genre de lravaux que l'aide de I'Etat est la plus saine, puisqu'il s'agit, par une aide essentiellement limitée dans le temps, de permettre à une population tout entière d'obtenir un meilleur rendement du travail, le plein emploi des hommes et des terres, l'élévation du niveau de vie et une activité générale accrue pour la nation.

Il y a des objections et l'on s'en aperçoit quand on prend l'avis des associations d'agri- 
culteurs, par exemple, ou quand on veut faire accepter une loi de finances par le Parlement. Il y a des adversaires.

On nie d'abord, du point de vue social, que les régions où les grands travaux sont possibles soient celles-là même qui méritent les plus gros efforts, tant par le niveau de vie de leurs habitants que par le chiffre de leur population.

On nie ensuite que l'augmentation de la production et de la productivité, d'emploi et de niveau de vie, soit nécessairement plus grande sur 1.000 hectares qu'on irrigue que sur 10.000 hectares qu'on recalcifie, par exemple. Autrement dit, on met en doute que la même somme dépensée en « grands travaux 》 soit toujours plus efficacement dépensée que si elle l'était de toute autre manière (recherche, mécanisation, engrais, etc., etc.).

Examinons d'abord la première objection qui est pour nous fondamentale, dans un cycle d'études consacré aux régions sous-développées.

Si l'on parle de régions dont la prospérité peut et doit être améliorée, les régions où de grands travaux sont possibles rentrent toutes dans la catégorie en question : l'irrigation, l'assèchement, la production d'électricité améliorent certainement la situation économique $\mathrm{du}$ pays. Mais ce serait prendre bien légèrement le problème : toutes les régions de France sont sousdéveloppées avec ce critère-là; partout une amélioration est nécessaire. Il n'en reste pas moins que dans les pays où de grands travaux sont réalisables, cette amélioration est possible, et c'est un point essentiel.

Une remarque s'impose, c'est que le transfert des populations est surtout redouté quand il s'agit d'exode vers les villes. Le transfert de campagne à campagne est un moindre mal. La seconde remarque, et qui vient immédiatement à l'esprit, c'est que les grands travaux ne sont possibles qu'en certaines régions du territoire national et que l'on n'a pas le choix de l'emplacement.

Ainsi les projets du Bas-Rhône et du Languedoc signalent-ils que l'irrigation est susceptible d'apporter des éléments d'activité nouveaux aux régions voisines de garrigues et de montagnes.

\section{NÉCESSITÉ D'ÉTUdES COMPLÈTES ET PRÉCISES :}

Une objection est faite, avons-nous dit, par les associations d'exploitants agricoles, par l'ensemble des habitants de la France et par leurs représentants, objection aux termes de laquelle il ne faut pas considérer comme évident qu'un grand ouvrage soit toujours plus utile qu'une multitude de petits et que la priorité doive être donnée aux grands travaux sur les améliorations locales.

Dans son principe, cette objection est raison- nable. On peut soupçonner, bien sûr, que des intérêts de « clocher », ce qu'il est convenu d'appeler des intérêts électoraux, aient le pas, dans cette tendance à l'atomisation des crédits, sur l'intérêt gẻnéral.

Mais il est exact que tous les « grands travaux $\gg$ ne sont pas bons à faire. Ils se comparent effectivement aux autres interventions possibles de l'Etat. II n'y a pas de choix a priori. On ne décidera qu'après des études qui auront permis les comparaisons indispensables. II faut des études très complètes, et systématiques sur tous les projets en présence, et dans le cas de grands travaux hydrauliques, changeant la mise en valeur d'une région, ces études sont très complexes. Elles sont lentes, elles sont coûteuses, elles demandent la collaboration de spécialistes divers et avertis. Il faut associer, dans l'esprit de tous, grands travaux et grandes études.

Ce sont ces études qui permettront de déterminer l'intérêt économique et social de l'aménagement régional proposé. Elles comporteront tous les éléments de la décision du choix gouvernemental. Elles permettront ensuite, si ce choix est positif, de mettre le projet à exécution, dans les meilleures conditions tant pour la réalisation des ouvrages eux-mêmes que pour l'exploitation des possibilités nouvelles offertes à la région.

La façon de mener les études est l'objet d'un des principaux chapitres de notre rapport. C'est un point absolument capital. Sans doute les indications que nous y donnons ne prétendentelles pas à l'originalité. Ces problèmes ont fait l'objet, ici et là, d'études très poussées et de réalisations remarquables. Il ne semble pas cependant entré dans les mœurs administratives, d'accorder à ces études préalables, toute l'importance qu'elles méritent. Il ne semble pas que l'organisation administrative soit toujours adaptée aux problèmes que la nature pose dans certaines régions avec une telle force que ce sont les structures administratives qui doivent se plier et non le problème naturel. Il semble qu'en fait, ces projets, dans telle ou telle région de France, aient été abordés par les plus diligents et selon des méthodes personnelles : l'improvisation est la force et la faiblesse de notre pays.

Les projets ainsi établis peuvent présenter des lacunes importantes, ils ne sont en tout cas pas comparables les uns aux autres et l'application d'une politique d'ensemble en est rendue plus difficile. On souhaite voir plus d'homogénéité dans ces études, on souhaite voir l'assèchement des marais de l'ouest étudié avec le même soin que l'irrigation du Languedoc et même on souhaite que toutes les études de ce genre soient faites avec toutes les garanties qu'un système bien étudié et passé en force de règlement peut seul assurer à tout coup. 


\section{II. - Rappel des grands travaux prévus au plan}

Les grands aménagements régionaux prévus par le deuxième plan d'équipement et de modernisation (1954-1957) tout en s'occupant du problème de l'énergie électrique — au moins pour la Durance - ont surtout pour but de modifier les conditions naturelles d'une région par des travaux d'irrigation et d'assainissement. Il est à noter qu'un certain nombre de membres des commissions de la production agricole et de l'équipement rural ont estimé que ces aménagements régionaux n'étaient pas « absolument nécessaires " à l'augmentation de $20 \%$ de la production agricole, chiffre fixé comme but ì atteindre par le plan. En outre, selon eux, l'appauvrissement du fait de l'arrachage de la vigne et le manque d'équipement hydraulique ne sont pas particuliers aux régions proposée'; mais s'étendent sur l'ensemble des territoires du centre, de l'ouest et du midi. Néanmoins, ils ont admis qu'il y aurait un problème social et humain qui nécessiterait « un effort de toute la nation ».

\section{PREVISIONS}

a) IRRIgation ET MISE EN VALEUR DU BASSIN DE: LA DURANCE: :

L'aménagement de cette région constitue unc source d'avantages:

- avantages industriels et fourniture d'énergic électrique (3 milliards de $\mathrm{kWh} / \mathrm{an}$ );

- avantages agricoles.

La mise en valeur agricole consiste essentiellement, d'une part dans l'extension et l'amélioration des périmètres irrigués de la basse vallée de la Durance (Vaucluse et Bouches-du-Rhône), d'autre part, dans l'irrigation des territoires du Var et des Bouches-du-Rhône, tributaires du Verdon et éventuellement dans la remise en valeur des régions de haute et moyenne altitude (Hautes-Alpes et Basses-Alpes), par la création, l'extension et la modernisation de réseaux d'irrigation à partir de la Durance et de ses affluents.

La construction du barrage de Serre-Poncon, dont une tranche de 200 millions de $\mathrm{m}^{3}$ sera destinée aux usagers agricoles, permettra :

- l'extension des irrigations sur 30.000 hectares;

- la conversion de $\mathbf{1 0 . 0 0 0}$ hectares de vignes en polyculture;

- l'amélioration des irrigations sur 70.000 hectares par la suppression des pénuries.
Le coût total d'établissement de l'ouvrage est actuellement évalué à 50 milliards, dont 6 milliards à la charge de l'agriculture.

L'existence de la réserve de Serre-Poncon facilitera l'irrigation des zones du Var, tributaires du Verdon. Ces travaux permettront l'irrigation de 25.000 hectares et assureront l'alimentation en eau de 80.000 personnes dans les Bouches-du-Rhône et de 23 communes dans le Var.

\section{b) BAs-Rhône et LANGUEdoc :}

La réalisation de ce projet doil permettre par l'établ:ssement d'un réseau d'irrigation, la donination d'un territoire de 160.000 hectares dans les départements de l'Hérault, de l'Aude et du Gard. 90.000 hectares pourront etre irrigués ainsi que 25.000 dans les vallées de l'Hérault et de l'Orb.

Les travaux permettront de :

- gagner 30.000 hectares à la culture (terres incultes);

- procéder à la conversion de 60.000 hectares de vignes en polyculture.

Le coût total des travaux atteindra 33 milliards.

\section{c) Coteaud de Gascogne:}

La mise en valeur de cette région consiste essentiellement à fertiliser par les caux d'irrigation en provenance du massif pyréncen et au besoin de la Garonne, l'ensemble de coteaux et vallées existant de part et d'autre des affluents de la rive gauche de la Garonne el de la rive droite de l'Adour dans leur cours supérieur.

Le périmètre de cette région couve 1.050 .000 hectares répartis sur les départements :

- du Gers . . . . . . . . 605.000 hectares

- de la Haute-Garonne. 215.000 hectares

- du Tarn-et-Garonne. $\quad 90.000$ hectares

-- du Lot-et-Garonne... 70.000 hectares

-des Hautes-Pyrénées. 70.000 hectares

La sécheresse y compromet les récoltes quatre années sur cinq. L'économic de la région esl essentiellement agricole. En un siècle, dans te seul département du Gers, la population a diminué de près de $40 \%$.

Le projet prévoit l'irrigation de 200.000 hectares, ce qui permettra une intensification considérable de la culture dans le cadre d'exploitations familiales modernisées.

- Production de fourrage et amćlioration de la viande et du lait,

- Production céréalière, 
- Culture maraichère et fruitière.

Le coût du projet s'èlève à 45 milliards.

\section{d) Amélioration des régions marécageuses DE L'OUEST :}

Les principaux marais dont la mise en valeur peut être envisagée sont :

- le marais bas-breton,

- le marais de la vallée du Lay,

-.- le marais de Luçon ou marais desséchés,

- le marais poitevin ou marais mouillés,

- le marais charentais.

En fait, l'effort du deuxième plan portera sur le marais poitevin qui a fait l'objet d'études préliminaires. Sur une superficie totale de 80.000 hectares, il a été décidé d'en assainir 17.500 dont :

- 5.500 hectares pour les Deux-Sèvres,

- 4.000 hectares pour la Charente-Maritime,

- 8.000 hectares pour la Vendée.

Les inondations menacent constamment cette région et depuis un siècle la population n'a cessé de décroître. Le projet vise essentiellement à faciliter au maximum l'écoulement des eaux. Le coût probable des travaux a été estimé environ à 1 milliard 300 millions.

Cet aménagement apporterait tout d'abord une augmentation substantielle de la production agricole (de l'ordre de 400 millions de francs) et améliorerait les conditions d'existence de plus de 3.000 familles et stopperait l'important exode qui sévit dans la contrée.

\section{e) Mise en valeur des landes de Gascogne:}

Les landes de Gascogne couvrent 1.400 .000 hectares et leur revenu s'élève à 15 milliards dont $1 / 3$ de produits agricoles forestiers.

Ce projet envisage la mise en culture de 50.000 hectares et la récupération après assainissement de 35.000 hectares de territoires marécageux. L'implantation de $\mathbf{5 . 0 0 0}$ familles est envisagée. Les surfaces gagnées à la culture permettront le développement de productions fourragères et de l'élevage.

L'ensemble des dépenses atteindrait 38 milliards.

\section{f) Afrique du Nord :}

L'Afrique du Nord a fait également l'objet de plan de mise en valeur par des travaux hydrauliques. Les problèmes d'aménagement et d'irrigation revêtent une exceptionnelle importance, en raison de la très forte pression démographique (400.000 hommes de plus par an). Il faut donc à tout prix mettre en valeur de nouvelles terres, l'émigration n'absorbant qu'une faible partie de l'excédent de la population qui dans cinquante ans aura presque doublé.

Dans cette région où l'irrégularité du climat et les caprices de la pluviométrie déterminent presque toujours le volume des récoltes, cette double action exige un effort parallèle d'équipement hydraulique, grands barrages ou travaux plus modestes mais dont la rentabilité peut être plus certaine et plus rapide : aménagements de petite et moyenne hydraulique.

Il paraît nécessaire d'insister sur l'aspect social essentiel du développement des irrigations en Afrique du Nord qui jonent le rôle de régularisateur des conditions atmosphériques. Elles contribuent à l'élévation du niveau de vie des populations rurales, condition même du succès des techniques agricoles perfectionnées.

Un hectare de cultures irriguées peut faire vivie une famille de six personnes, compte tenu du niveau de vie actuel.

Autre problème lié à l'hydraulique agricole celui de la restauration des sols. A quoi bon construire des barrages, si la lutte contre l'érosion des terres arables n'est pas entreprise?

\section{AlgÉrie}

En Algérie, les barrages existants dominent un ensemble de périmètre de 170.000 hectares dont 89.000 sont irrigables et 45.000 étaient effectivement irrigués en 1953. II semble que cette politique de grande hydraulique n'ait pas donné encore tous les résultats escomptés du fait de la lenteur de l'équipement des périmètres qui restent trop longtemps inachevés. Il a été demandé au cours de l'exécution du $2^{\circ}$ plan quadriennal (1953-1956) de faire porter l'effort :

- Sur l'achèvement de l'équipement des périmètres dominés par les barrages existants et sur l'aide aux propriétaires pour leur permettre par des prêts à moyen et long termes, d'aménager leurs terres en vue d'une irrigation rationnelle;

- Sur la petite et moyenne hydraulique par :

- l'alimentation en eau des populations;

- l'équipement des zones favorables à l'élevage (points d'eau, puits, captage de source et développement de cultures fourragères);

- mise en valeur des terres dans les territoires du Sud par barrages de dérivation et forages de puits artésiens;

- Sur la lutte contre les eaux nuisibles.

Un crédit de 8 inilliards par an est inscrit au budget. 


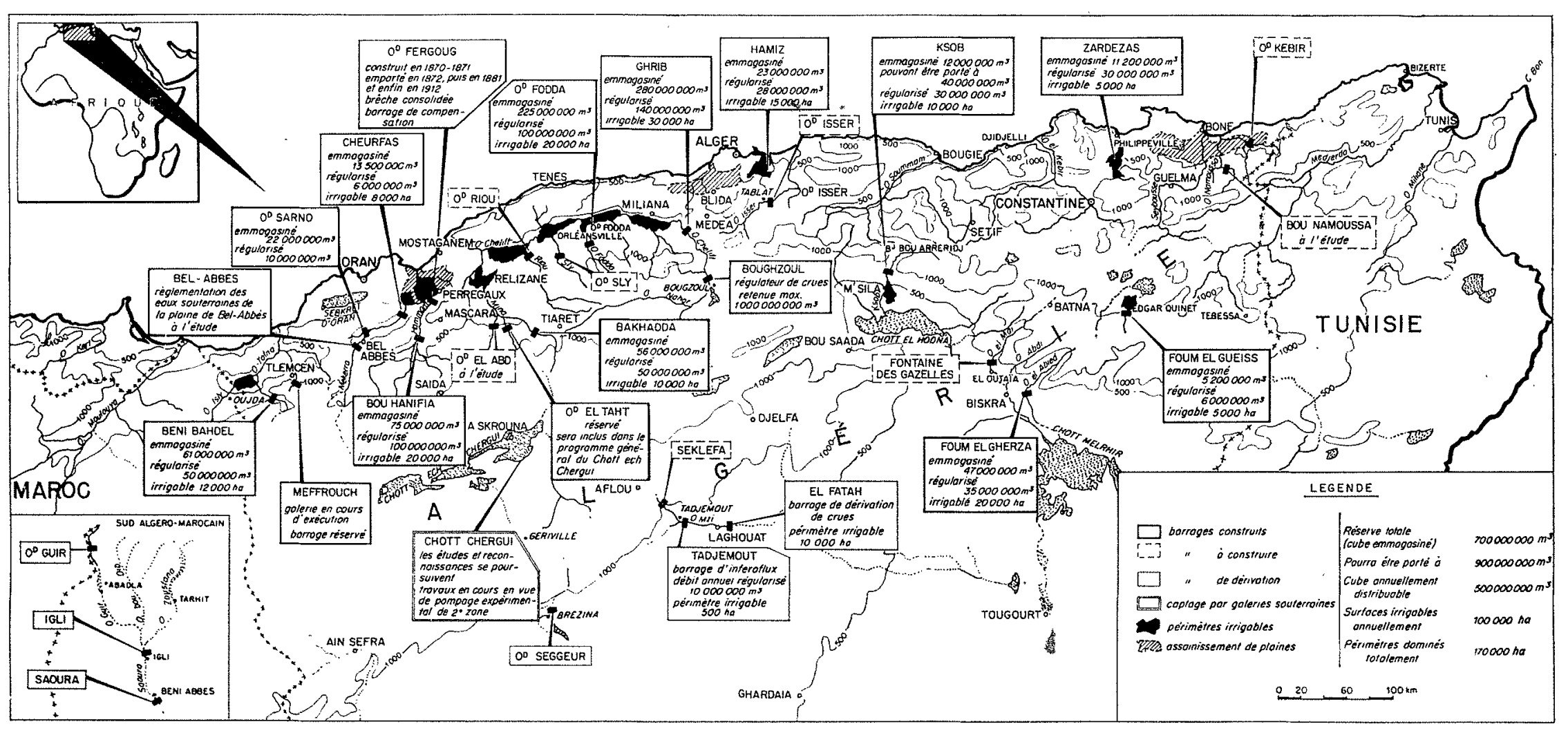

Fig. 1. - L'Algérie hydraulique. 


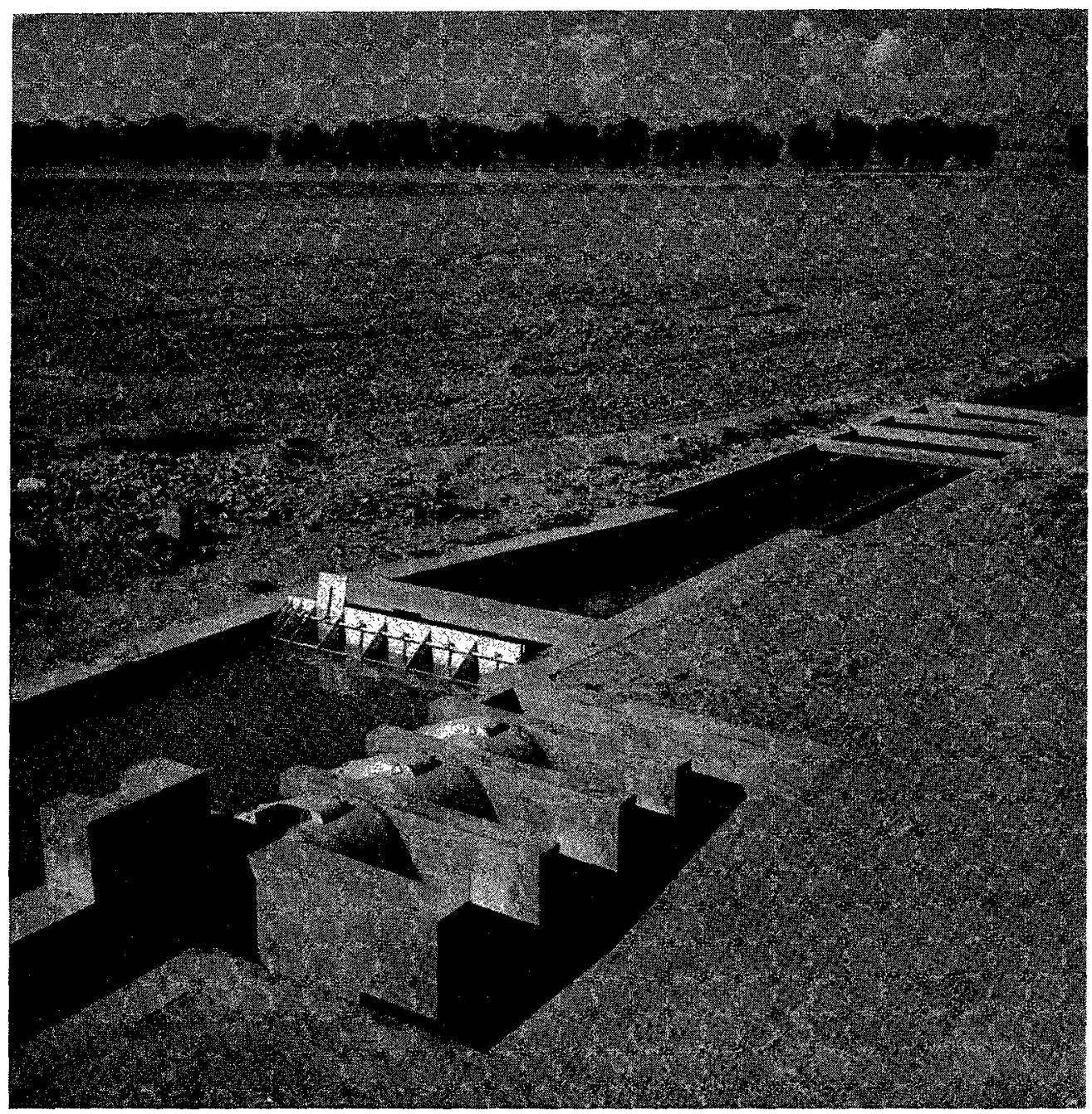

FIG. 1 bis. - Partiteur à Relizanc.

Maroc

Actuellement, l'irrigation au Maroc se pratique sur plus de 200.000 hectares. L'achèvement du plan en cours d'exécution permettra l'irrigation de 800.000 hectares dont près des $2 / 3$ en irrigation permanente.

Comme en Algérie, les travaux de petite et moyenne hydraulique sont menés en concomitance avec la politique des grands barrages.

Le deuxième programme quadriennal de moyenne et petite hydraulique doit porter sur l'irrigation de 60.000 hectares, représentant une dépense de 7 milliards, dont 5.658 millions subventionnés par l'Etat et 1.300 millions provenant de l'apport des utilisateurs ou de prêts consentis par les organismes de crédits agricoles.

En matière de forage, d'équipement pastoral et d'adduction d'eau, il est également prévu :

-25.000 à 30.000 mètres de forage,

- l'aménagement d'un millier de points d'eau et la constitution de réserves,
- un ensemble d'adduction d'eau permettant d'alimenter 50.000 personnes ou $\mathbf{5 0 0 . 0 0 0}$ têtes de petit bétail.

Les crédits prévus s'élèvent à plus de deux milliards, dont la presque totalité est à la charge de l'Etat.

Quant à l'ensemble des grands périmètres en cours d'organisation, qui sont au nombre de six, il porte sur 400.000 hectares.

En dehors du coût des barrages entièrement financé par l'Electricité, l'effort financier est de l'ordre de cinq milliards par an.

Le plan 1953-1957 s'est limité à l'équipement des périmètres de :
Triffas.......
8.000 hectares
Sidi Slimane.
7.500 hectares
Doukkalas....
9.700 hectares
Beni-Moussa. .
14.000 hectares

\section{Tunisie}

En matière de grands travaux hydrauliques, les efforts du plan sont concentrés sur la mise 


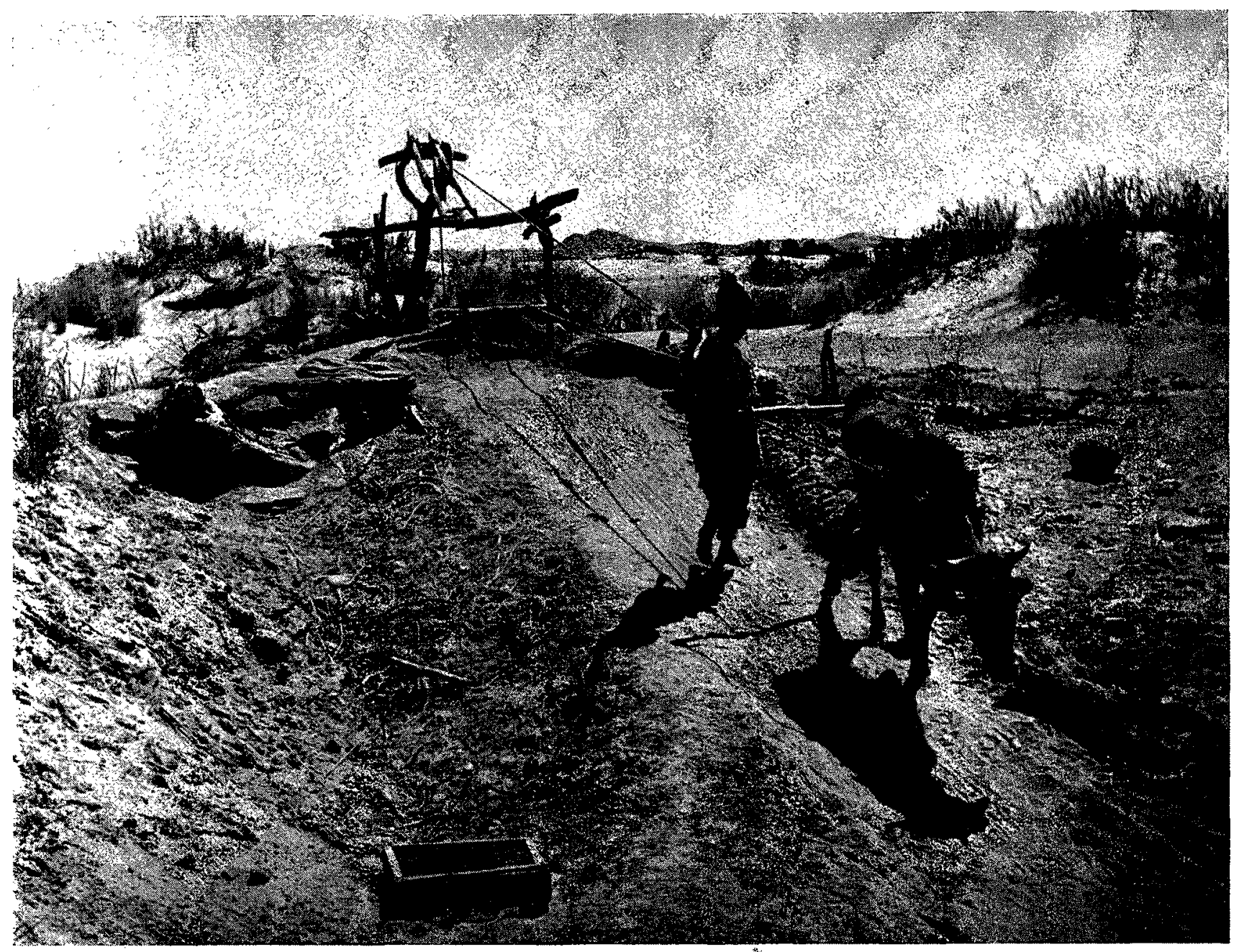

(lholo I. Brin.)

lis. 2. - « Delou », puits primitif dans le Tafilalet. L'eau remonléc dans une outre par traction animale ou humaine est ensuite déverséc dans une séguia d'alimentalion.

en valeur de la vallée de la Medjerda, où une superficie de 35.000 hectares est susceptible d'irrigation par l'équipement de deux barrages qui permettront l'alimentation en eau de la ville de Tunis et la production d'énergie hydroélectrique (90 millions de $\mathrm{kWh}$ ).

\section{g) France d'Outre-Mer :}

Les grands projets d'aménagements hydrauliques du plan 1953-1957 intéressant la France d'Ontre-Mer couvrent 140.000 hectares et coûtent 40 milliards. Ils sont relativement nombreux :

-- Extension des zones irriguées de l'Office du Niger (42.000 ha);

- Aménagement de la vallée du Sénégal et plus particulièrement secteur de modernisation agricole de Richard Toll $(6.000 \mathrm{ha})$;

- Haute Volta (14.000 ha). - Guinée (18.000 ha);

- Complexes hydroélectriques de Guinée (grandes chutes : $45 \mathrm{MkWh}$ - Koukouré :
$5.000 \mathrm{M} \mathrm{kWh}$ ), du Cameroun (Edéa : $900 \mathrm{M} \mathrm{kW}$ ) et du Congo (Djouć : $100 \mathrm{M}$ $\mathrm{kWh}$;

- Projet d'aménagement des vallées du Chari et du Logone au Tchad (5.000 ha extensibles à plusieurs centaines de mille);

- Aménagement des vallées de Ja Mahavavy et du Sambarino (Sosumav : 10.000 ha par société privée), secteurs de riziculture de Marovoay et du lac Alaotra à Madagascar (45.000 ha); etc...

En outre, plusieurs aménagements de centaines de milliers d'hectares sont à l'étude : Sénégal, Niger, Haute Volta, Dahomey, Cameroun, Tchad, Madagascar, Guinée...

Ne pouvant dans le cadre de notre étude procéder à l'examen même sommaire de l'ensemble de ces projets ou réalisations, nous nous bornerons à une description sommaire des deux premiers.

L'Office du Niger, constitué en 1932, est essen- 


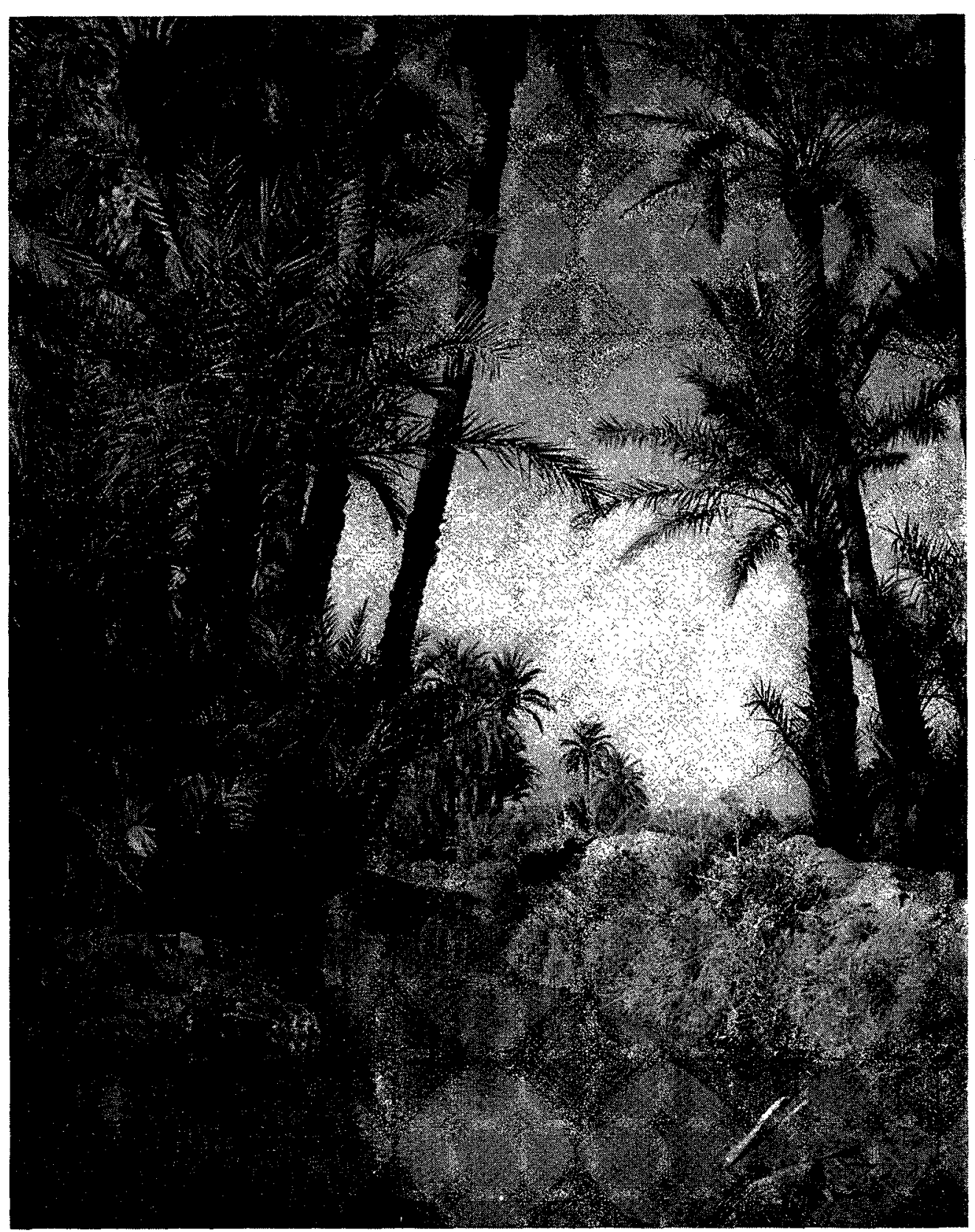

Fig. 2 bis. - Partie de palmeraie de Marrakech irriguée par la séguia Azouzia.

tiellement consacré à la mise en valeur du Delta central nigérien, le Delta vif (actuellement en eau) devant après aménagement être consacré à la riziculture inondée, le Delta mort (anciens affluents desséchés) étant destiné après remise en eau et irrigation à la culture du coton.

Le projet initial comportait l'aménagement de 510.000 hectares de terres à coton et de 450.000 hectares de rizières, soit au total 960.000 hectares. Il devait permettre l'installation de 300.000 colons africains. La dépense était évaluée à environ 300 millions de franes 1929.

L'équipement de base actuellement terminé a pour pièce mâtresse le barrage de Sansanding, large de $2.600 \mathrm{~m}$, dont une partie mobile de $816 \mathrm{~m}$ équipée de vannes à hausse; il permet d'élever le plàn d'eau à $5 \mathrm{~m}$ au-dessus des eaux moyennes et est doublé par un canal de navigation de $8 \mathrm{~km}$ de long permettant de passer d'un bief à l'autre.

$260 \mathrm{~km}$ de grands canaux et endiguements, $1.900 \mathrm{~km}$ de canaux principaux et $1.000 \mathrm{~km}$ de rigoles de drainage et diguettes ont été aménagés.

Le coût des travaux effectués de 1926 à 1952 évalués en francs actuels métropolitains s'élève à 9 milliards. 


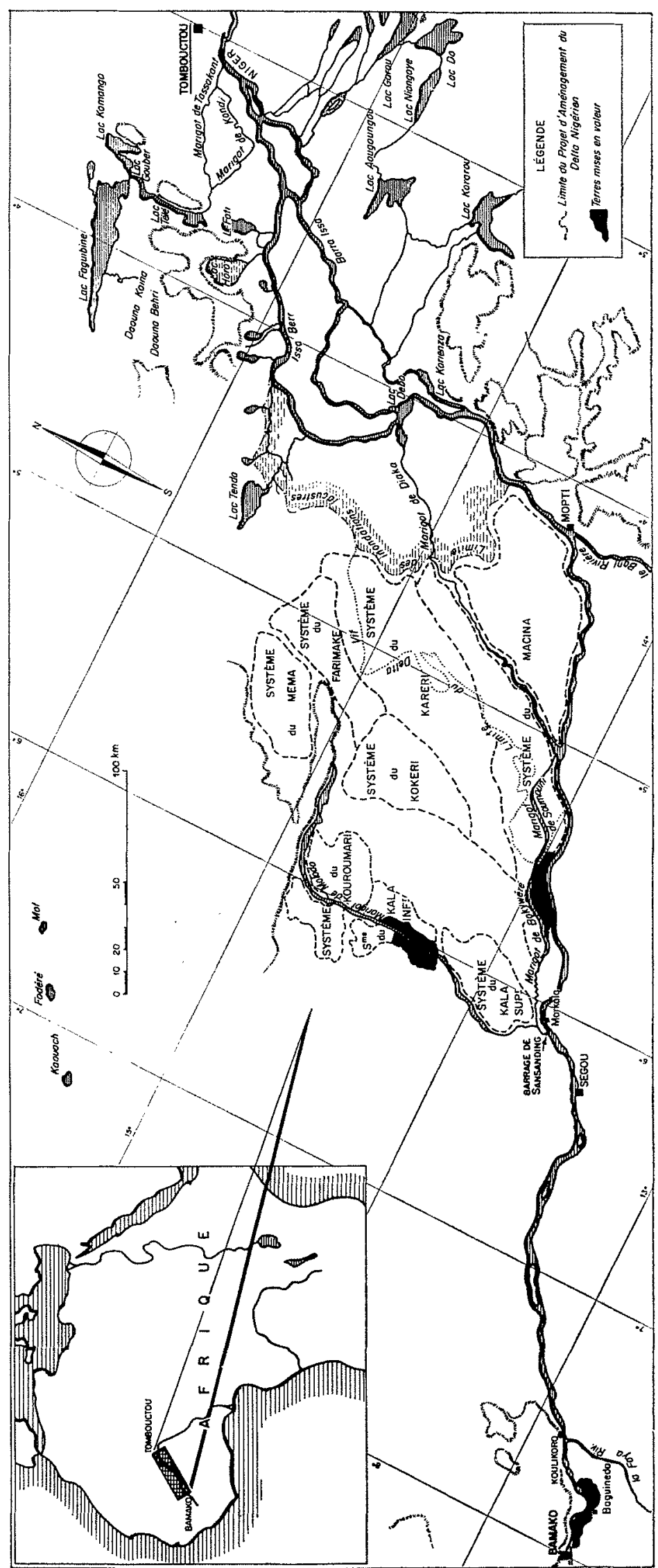

Fig. 3.

Garte de la vallée du Niger de Bamako à Tombouctou.

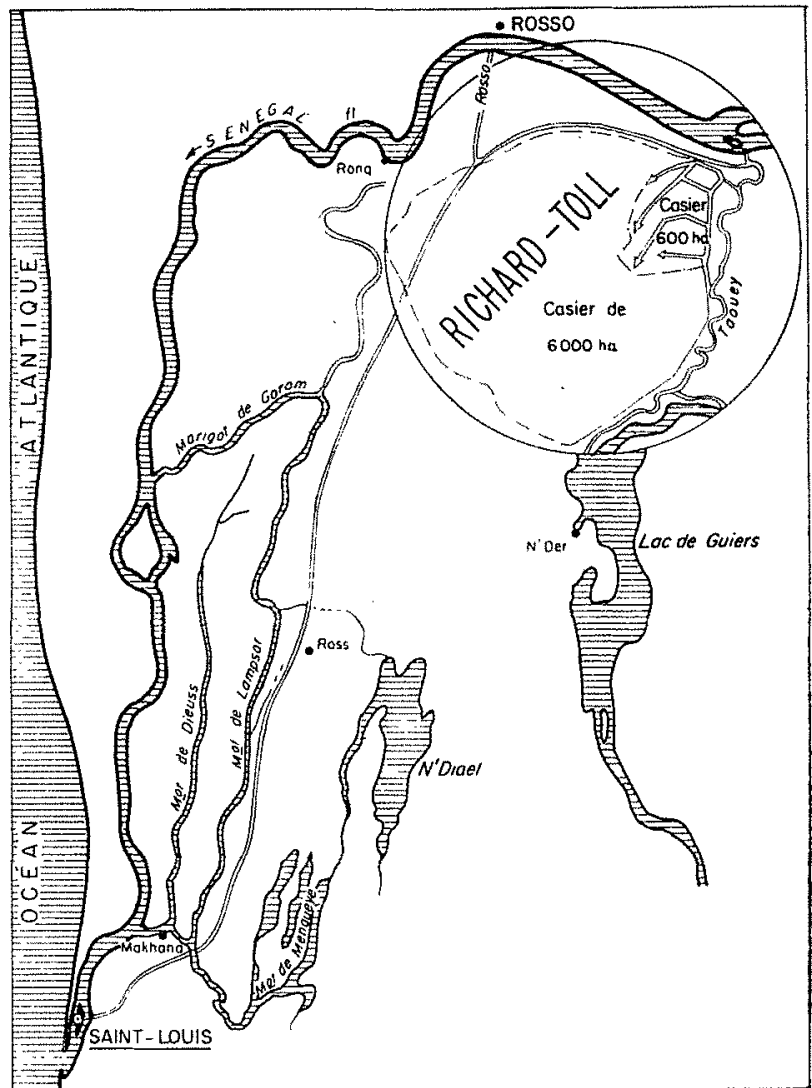

Fig. 4. - Casier de 6000 ha à Richard-Toll. Plan schématique de situation.

En 1953-1954, les superficies cultivées étaient évaluées à 38.344 ha et la récolte à $40.000 \mathrm{t}$ de paddy et $3.500 \mathrm{t}$ de coton.

Des extensions sont prévues sur le plan quadriennal en cour's qui, primitivement fixées à 50.000 ha, ont été ramenées à 20.000 ha dans la zone cotonnière et 6.000 ha dans la zone rizicole.

Il est évident que ces superficies sont encore infimes par rapport au projet initial et qu'elles n'ont aucune commune mesure avec l'importance de l'infrastructure réalisée, ce qui explique en partie les difficultés financières d'exploilation.

Richard-Told, dans la zone du delta du fleuve, est la première étape d'un plan général d'aménagement de la vallée du Sénégal actuellement à l'étude. Les travaux sont conduits par la Mission d'Aménagement du Sénégal (M.A.S.), organisme groupant des techniciens des services des Travaux publics et de l'Agriculture et rattaché au Service des Travaux publics du Sénégal. II a également été fait appel pour certains travaux à des entreprises privées, 


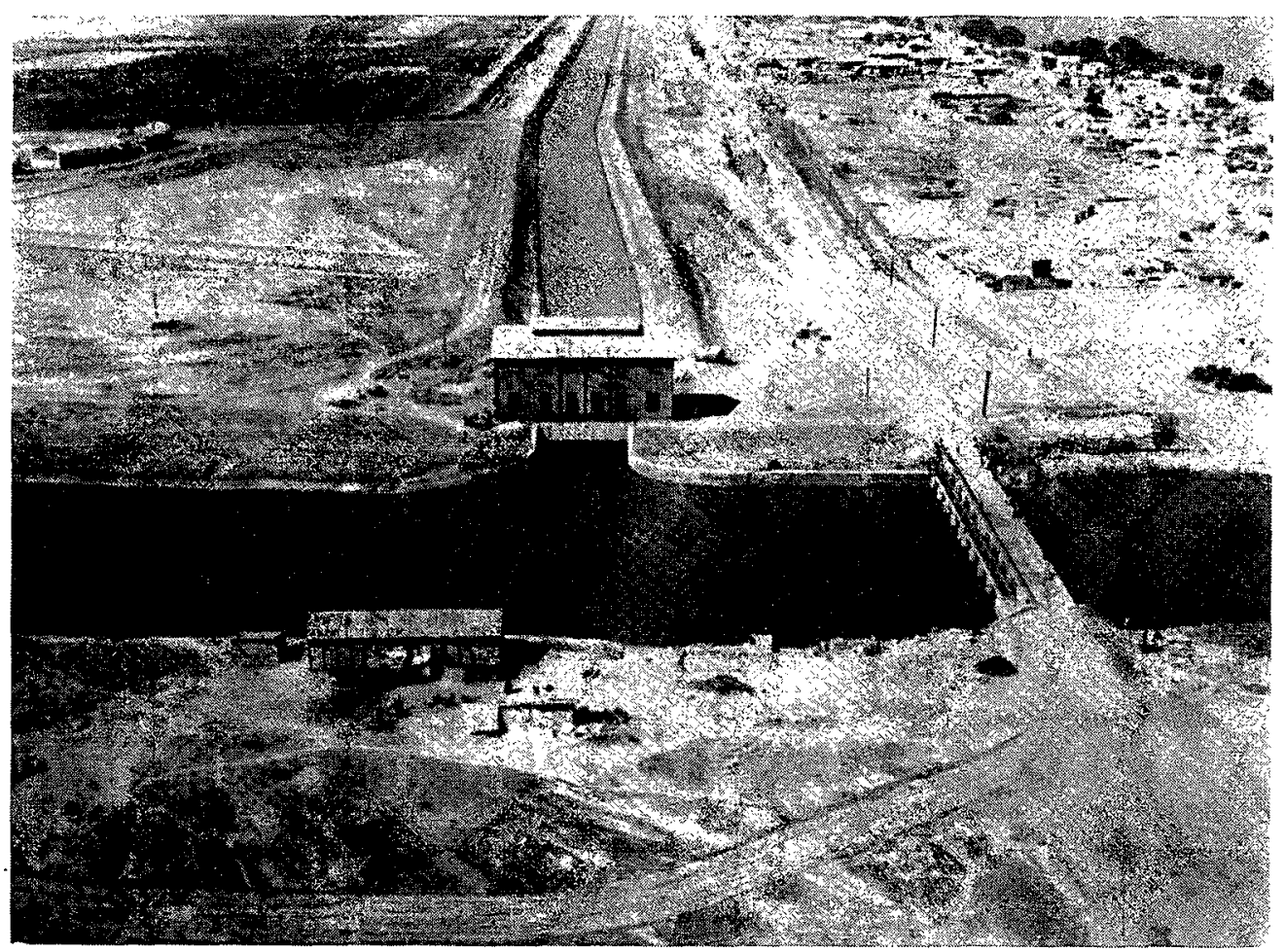

Fig. 5. - Richard-Toll : station de pompage du casier de 6000 ha et pont-barrage sur la Taouey.

Dès 1945, un casier expérimental de 120 ha était créé qui a été porté d'abord à 600 ha puis à 6.000 ha et exploité sous la direction d'un ingénieur des services agricoles.

Les aménagements comprennent :

- Le barrage de la Taouey destiné à créer une réserve d'eau douce dans le lac de Guiers, réserve destinée à l'irrigation et au dessalage des terres;

- Une rectification du lit de la Taouey et un endiguement du lac;

- Le pompage des eaux d'irrigation à RichardToll;

- Un canal principal de $50 \mathrm{~km}$ avec station d'exhaure sur le parcours;

- Le drainage par gravité d'une vaste dépression salée.
Le coût des aménagements est de l'ordre de 2 milliards qui ont été financés par le F.I.D.E.S. Leur achèvement et la mise en valeur de tout le lotissement nécessiteront 700 millions supplémentaires. Ces aménagements pourront servir pour la création d'un second casier de 6.000 ha prévu. Ils sont complétés par diverses installations (parc de gros matériel, rizerie, station de pompage, atelier, quai, centres administratif et résidentiel, etc.). Les possibilités d'extension sont de l'ordre de 20.000 à 50.000 ha.

Il s'agit essentiellement d'un centre expérimental de riziculture mécanique destiné à combler le déficit alimentaire du Sénégal, pays de monoculture de l'arachide. Le rendement moyen est de l'ordre de 27 quintaux par hectare. La dernière récolte a été de $3.600 \mathrm{t}$. On espère porter rapidement cette production à $15,000 \mathrm{t}$ sur le seul premier casier de 6.000 ha. 
DEUXIEME PARTIE

\section{CONDUITE DES ETUDES}

\section{I. - Eléments à étudier}

L'exécution de grands travaux d'hydraulique doit être précédée de longues études qui doivent porter sur un grand nombre d'éléments techniques, économiques et sociaux.

Ces études sont onéreuses, mais malgré l'impatience que l'on peut avoir de passer rapidement à l'exécution de certaines parties de l'aménagement, il est indispensable qu'elles soient complètement menées pour que toutes les conséquences, présentes et futures, aient été examinées avant qu'on accorde les crédits des travaux et qu'on décide de passer à l'exécution.

\section{a) Eléments hydrauliques, topographiques et CLIMATOLOGIQUES :}

L'essentiel des travaux d'hydraulique consiste à amener de l'eau là où l'on en manque, à se protéger contre les arrivées d'eau intempestives et à évacuer les eaux stagnantes. Il faudra done d'abord étudier le climat pour connaitre les probabilités et l'importance des pluies. Il faudra ensuite mener des études hydrologiques sur les différentes rivières dont on envisage de prendre l'eau ou contre les crues desquelles on veut se protéger. Ces études hydrologiques devront couvrir tout le bassin situé en amont des ouvrages de prise d'eau et devront comprendre également le cours de la rivière situé en aval, de façon à prévoir quelles seront les conséquences sur cette rivière et sur les usages qui y sont faits de l'eau, des modifications de régime entraînées par les ouvrages.

L'étude hydrologique devra également comporter des analyses de l'eau prélevée donnant la quantité de matières solides et leur composition, ce qui peut être très important en matière de limonage des terres et d'envasement des retenues. Il faudra tenir compte d'ailleurs des modifications à ces teneurs en matières solides qui résulteront du passage éventuel dans des retenues tranquillisées. La teneur en sels dissous est importante pour prévoir l'enrichissement éventuel des terres irriguées ou au contraire leur empoisonnement progressif.

Bien entendu, une étude des débits comparés de la rivière et des besoins de lirrigation aux différentes époques de diverses années devra être menée, en vue d'établir, par avance, un projet de programme d'exploitation des ouvrages.

L'étude de l'eau ne doit pas être limitée à ce qui se passe en surface. En effet, il peut arriver que l'irrigation soit assurée à partir d'eaux souterraines et il peut arriver également que les modifications au régime des eaux souterraines résultant des ouvrages de retenue ou de l'irrigation proprement dite aient des conséquences importantes pour le voisinage.

Enfin, il est bien évident qu'une utilisation rationnelle de l'eau, avec des ouvrages aussi économiques que possible, ne pourra pas être réalisée sans une étude topographique complète couvrant aussi bien l'ensemble des périmètres à aménager que la retenue en amont des ouvrages.

Une étude comparée du débit prélevé et du débit restitué à la rivière permettra d'évaluer les dommages aux personnes situées en aval et également les avantages que celles-ci obtiendront d'une plus grande régularité du débit.

Lorsqu'il s'agira de travaux d'assainissement, il faudra conserver présent à l'esprit le fait que l'amélioration de l'écoulement dans une région a pour conséquence la disparition de l'effet régulateur qu'avaient les marais sur les crues. Un tel assainissement aggravera donc forcément la situation des riverains d'aval et l'irrégularité du débit de l'exutoire. Il arrivera donc que des travaux d'assainissement prévus en un endroit déterminé aient pour conséquence l'aménagement de l'évacuation sur de très grandes longueurs à l'aval.

Lorsqu'il s'agira de défendre certains terrains contre l'envahissement par les crues, il ne faudra pas oublier qu'une telle défense a généralement pour conséquence d'aggraver l'importance des crues au droit des digues et sur toute la partie amont. Elle a également pour inconvénient de supprimer, dans les terres mises en culture, l'apport d'éléments fertilisants qui résultait de leur submersion.

L'étude hydraulique et topographique comparée permettra de choisir entre les solutions d'irrigation et drainage par gravité et les solutions par pompage qui nécessitent des frais annuels plus considérables, 


\section{b) Eléments agronomigues FT pédologiQues :}

Il ne faut pas oublier, lorsqu'on étudie un programme d'hydraulique agricole, que l'objet essentiel du programme est d'améliorer l'agriculture, tandis que la maîtrise de l'eau n'est qu'un des moyens d'obtenir cette amélioration.

Toute étude d'un tel projet devra donc comporter à la base non seulement l'élément hydraulique, mais encore et tout d'abord une carte pédologique complète classant tous les sols intéressés d'après leurs possibilités culturales et leurs perspectives d'évolution après irrigation.

Une étude agronomique devra exploiter cette carte pédologique, pour évaluer les possibilités des récoltes, compte tenu de la nature du sol et de la quantité de l'eau dont on dispose ainsi que du climat et pour essayer de déterminer quelles seront les dépenses d'engrais nécessaires aux diverses cultures. Dans bien des cas, il sera impossible de mener par avance une étude agronomique complète et l'on sera conduit à installer à proximité immédiate ou sur le lieu même des travaux envisagés une station agronomique permettant de mettre au point les facons culturales et chercher les espèces qui conviennent le mieux à la nature du sol, à la composition de l'eau et au climat.

Lorsque l'étude du sol aura montré la présence dans celui-ci d'éléments nuisibles, l'étude agronomique devra préciser quelles sont les quantités d'eau de lessivage à prévoir. Elle devra indiquer également, dans tous les cas, quel est le niveau souhaitable de la nappe phréatique pour les cultures envisagées, ce qui fixera la po'itique de drainage.

L'étude agronomique permettra de préciser jusqu'à quel degré de finition devra être réalisé l'aménagement de détail et notamment le planage des parcelles. Elle permettra également de donner les éléments du Règlement d'Eau qu'il faudra inclure parmi les règles d'exploitation ultérieures.

\section{c) ElÉments Garactéristiques De L'IRRIGATION ET DE L'ASSAINISSEMENT :}

Une étude rationnelle de l'hydrodynamique des sols, c'est-à-dire des rapports complexes entre le sol et l'eau, devra être entreprise dans tous les cas; elle permettra de déterminer les caractéristiques de base des projets d'irrigation et d'assainissement.

Les besoins en eau et les méthodes d'adminis. tration des arrosages devront être prévus à l'avance avec le plus de précisions possibles, pour ajuster les dimensions de l'aménagement aux besoins de la rég:on; on évitera ainsi le gaspillage de l'eau ou le gaspillage des investissements.

Enfin, les possibilités de dégradations physi- que, chimique et bactériologique des sols à irriguer devront être supputées par comparaison aux observations qui auront pu être faites sur des sols déjà irrigués, et qui ont des profils pédologiques analogues.

Il n'est pas inutile de dégager ici certains aspects caractéristiques de ces questions.

L'étude de l'irrigation devra déterminer :

- D'une part, les besoins en eau du complexe 《sol - plantes » et la variation de ces besoins aux diverses époques de l'année (moyennes mensuelles);

- D'autre part, le mode d'administration des arrosages (doses - fréquences - modules) qui dépend de la structure physique des sols (vitesse de filtration, coefficient de perméabilité).

$1^{\circ}$ Pour le premier point, il convient de notur que dans l'état actuel des connaissances des spécialistes, c’est la méthode américaine Braney et Criddle qui est couramment utilisée; cette méthode a été adaptée en France au système métrique et transformée en abaques d'utilisation commode par la Section Technique de l'Irrigation et de l'Assainissement. Dans cette étude entrent en ligne de compte trois paramètres: la plante, les températures moyennes mensuelles et les durées d'éclairement; on en déduit les besoins en eau pour compenser «l'évapo-trans. piration physiologique de la plante » (les besoins en eau de constitution des tissus végétaux sont négligeables, égaux à la différence entre l'évapotranspiration et les pluies qui tombent naturellement dans la région pendant la période de végétation).

$2^{\circ}$ Les doses d'arrosage et leurs fréquences sont fonction des caractéristiques hydrodynamiques des sols, et notamment du coefficient de perméabilité et de la vitesse de filtration; chaque sol peut, à ce point de vue, être caractérisé par :

-. Sa capacité de rétention, c'est-à-dire la quantité d'eau qu'il peut emmagasiner sans qu'il y ait perte par infiltration dans le sous-sol;

- Son «point de flétrissement», e'est-à-dire la quantité d'eau que les forces de succion des racines des plantes ne peuvent enlever au sol;

- Sa capacité utile en eau pour les plantes, qui est la différence entre les deux grandeurs ci-dessus.

La dose d'arrosage devra satisfaire la capacité de rétention, sans plus. La fréquence ou l'espacement entre deux arrosages correspond à l'absorption de la « capacité utile » par l'évapo-transpiration. 
La dose d'arrosage peut être administrée sur une surface donnée avec une durée plus un moins grande en rapport avec la vitesse de filtration dans le sol; c'est ce qui détermine le «module» ou «débit effectif 》d'arrosage; le calcul théorique montre que ce module pourrail varier de quelques litres à la seconde pour des unités parcellaires d'un demi-hectare dans des terrains très peu perméables, à 500 litres par seconde dans des terrains très perméables.
Les éléments caractéristiques de l'irrigation auxquels aboutiront les études devront donc faire apparaitre pour chaque zone ou casier homogène, et pour chaque culture envisagée :

1. Le débit fictif continu d'arrosage qui correspond aux besoins réels des plantes et qui peut varier avec la culture et le mois considérés, de 0,3 à 1,5 litre par hectare et par seconde.

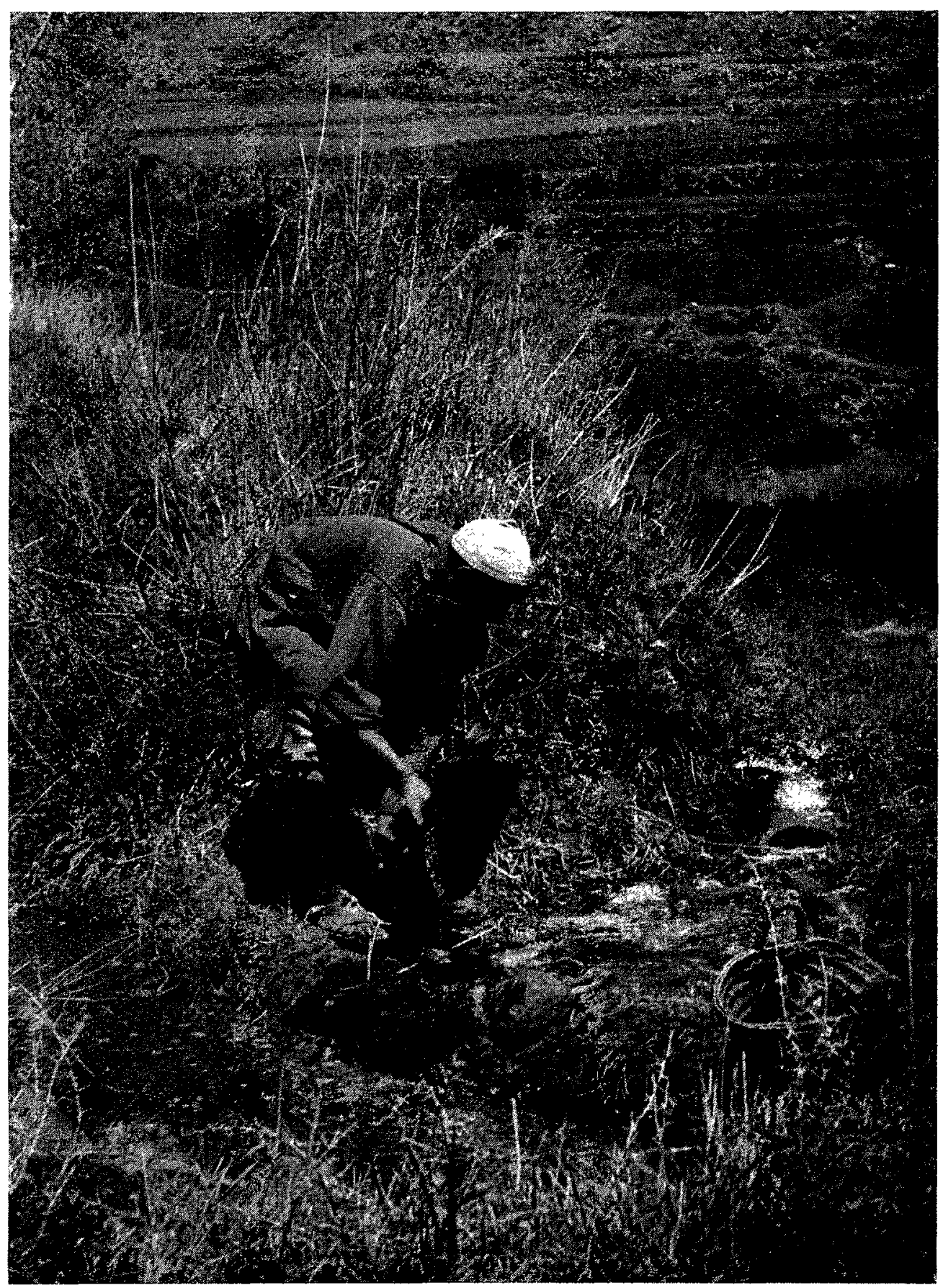

Fig. 6. - Irrigation dans les montagnes du Haut-Atlas, suivant la méthode traditionnelle des indigènes. 
2. La dose d'arrosage qui est la quantité d'eau que l'on amène en un temps limité sur les parcelles à irriguer; elle est fonction de la perméabilité du sol; elle doit être assez abondante pour pouvoir humecter toutes les couches accessibles aux racines.

3. Le module ou débit effectif d'arrosage qui dépend de la vitesse d'infiltration et de l'unité parcellaire d'arrosage.

Les mesures ou observations des caractéristiques hydrodynamiques des sols sont également à la base des études des projets d'assainissement et de drainage et permettent de déterminer les espacements, profondeurs et calibres des fossés ouverts ou des drains enterrés, en fonction de l'abaissement souhaitable du niveau des eaux pour les cultures envisagées.

\section{d) DÉFENSE DES SOLS ET AVENIR TECHNIQUE :}

II ne suffit pas que les résultats soient satisfaisants lors de la mise en exploitation des casiers. Il faut encore que les ouvrages conservent leur utilité pendant de très longs délais et que les sols mis en culture ne se dégradent pas. La première condition pourra être convenablement respectée en particulier si, le cas échéant, il est pris un certain nombre de mesures dans le bassin d'alimentation, telles que reboisement, cultures arbustives, banquettes, ouvrages de corrections des torrents, etc... dont l'effet sera d'éviter l'envasement des barrages. En outre, des dispositifs techniques concernant la prise d'eau permettent, lorsqu'on a eu l'attention attirée sur ce point, de prélever les eaux dans une zone où elles sont particulièrement chargées, ce qui présente le double intérêt de limiter l'envasement et de répandre des limons sur les zones irriguées.

L'autre aspect de la question concerne ce qu'on appelle la géomorphologie dynamique, c'estä-dire l'évolution des sols lorsque les conditions naturelles, auxquelles ils étaient soumis avant l'aménagement, sont modifiées, soit par les façons culturales, soit par le régime des eaux qui traversent ces sols.

\section{e) Autres éléments techniques :}

Un grand aménagement hydraulique non seulement intéresse souvent d'autres activités que l'agriculture, mais encore nécessite pour le plein succès du programme agricole, un certain nombre de mesures autres que les façons culturales et la maîtrise de l'eau. Dans le cadre des premières considérations, nous citerons d'abord la production de l'électricité d'origine hydraulique, qui est un des éléments essentiels de la rentabilité des ouvrages lorsque la constitution de réserves entraîne une chute de niveau appré- ciable. En principe, l'électricité ne consomme pas l'eau, elle la rend, mais à une cote et à une époque qui peuvent ne pas convenir à l'agriculture. Cependant, des époques sont bien préférables à celles des crues naturelles. Dans ce dernier cas, il y aurait lieu d'examiner s'il n'est pas possible de produire l'électricité par voie hydraulique à un rythme qui convienne mieux aux besoins agricoles et de satisfaire les besoins des consommateurs d'électricité au moyen de l'interconnexion et des centrales thermiques ou bien de réserver à l'agriculture une certaine tranche de retenue.

La navigation est également une des activités qui peuvent bénéficier de l'aménagement hydraulique, parallèlement à l'agriculture. En effet, la régularisation du débit d'un cours d'eau est toujours un élément favorable.

Quant à la pêche, il est rare qu'elle bénéficie des ouvrages, mais la législation prévoit que ses intérêts doivent être protégés.

Quant aux éléments techniques du projet qui doivent contribuer à la meilleure réalisation du programme et au plus grand bénéfice des cullivateurs, nous citerons tout d'abord les dessertes routières et l'industrie des matériaux de construction : briqueteries, tuyaux de drainage et d'adduction, etc... Enfin, le projet devra examiner et réserver les possibilités d'installation d'industries agricoles complémentaires du programme. Outre les installations de stockage qu'il faudra toujours prévoir, ces industries sont multiples : industrie mécanique de réparation d'engins mécaniques, rizeries dans le cas du riz, moulins pour le blé, frigorifiques pour les denrées périssables, confitureries pour les fruits, installations de rouissage pour les fibres textiles qui demandent ce traitement, éventuellement fabriques d'engrais chimiques, etc...

\section{f) Etude Économique :}

Ayant en mains le projet chiffré, tant en ce qui concerne le coût de construction que le coût d'exploitation et connaissant les possibilités agronomiques, il sera possible d'entamer l'étude économique. Elle comportera d'ailleurs deux genres de préoccupations bien distinctes: d'une part, une étude de la productivité des investissements, c'est-à-dire la comparaison du coût de ces investissements avec la valeur totale des productions attendues, supposées vendables à leur prix de revient, d'autre part, une étude de rentabilité budgétaire permettant d'évaluer comment la personne physique ou morale qui aura en charge les travaux de mise en valeur et les frais d'exploitation ultérieurs pourra faire face à ses charges.

En matière agricole, la partie la plus importante est d'ailleurs la première, car, étant donnć 
les difficultés d'établir une assiette convenable d'impôts ou de taxes sur les activités agricoles, on pourra être amené assez fréquemment, sinon à mettre à la charge de la puissance publique les frais d'exploitation, ce qui serait extrêmement dangereux, tout au moins à laisser définitivement à sa charge une partie plus ou moins importante du coût de premier établissement.

L'étude économique générale concernant l'avenir de la production est extrêmement difficile et bien souvent on n'ose pas s'y attaquer. Mais cette difficulté devra être au contraire une raison pour essayer de traiter la question dès l'origine des études, c'est-à-dire dès que les connaissances des ressources en eau et des sols permettent d'avoir une idée des diverses produetions possibles et de leur quantité; il y aura tout d'abord à étudier le marché des divers produits. Compte tenu des besoins en quantités du marché local, du marché national et du marché mondial et compte tenu également des variations du prix des denrées agricoles d'une année sur l'autre dans ces divers marchés, il faudra essayer d'évaluer à quel prix moyen la production agricole brute correspondant aux diverses vocations du sol pourra être achetée au cultivateur.

Il faudra d'autre part essayer de déterminer un prix de revient de la production en année moyenne qui tienne compte d'une part du rendement probable à l'hectare, d'autre part des prélèvements sur la récolte pour la subsistance du cultivateur et de sa famille, cette subsistance correspondant au niveau de vie avant aménagement, et enfin des diverses dépenses que doit payer le cultivateur. Ces dernières ne tiennent pas compte des taxes et redevances diverses qu'il peut être amené à verser à l'organisme chargé du périmètre d'irrigation pour amortissement des investissements; par contre, celles relatives aux frais d'entretien et d'exploitation doivent être comprises.

La comparaison de ce prix de revient minimum et du prix de vente moyen possible permettra d'envisager l'avenir avec plus ou moins de confiance, de se rendre compte si la production de tel ou tel produit doit rester très limitée, d'apprécier s'il y a des possibilités de déversement sur le marché local à prix élevé ou, au contraire, s'il est possible de vendre au prix mondial.

Si les prix de vente ainsi calculés pour les quantités prévues sont supérieurs aux prix de revient indiqués plus haut, l'affaire doit être considérée comme productive au point de vue économique et une partie de la marge peut être affectée à l'amortissement des investissements pour contribuer à la rentabilité budgétaire.

L'étude de cette dernière peut elle-même être conduite de plusieurs façons : la plus sage consiste à considérer l'organisme chargé du péri- mètre comme un emprunteur qui a reçu des fonds frappés d'un taux d'intérêt normal et amortissable en une certaine durée. II aura done comme dépenses d'une part les charges financières résultant de cet emprunt, d'autre part les charges d'exploitation courante et les charges de renouvellement des parties périssables de l'aménagement (pompes, moteurs). Comme recettes cet organisme aura d'abord le prix de vente de l'eau, il pourra ensuite percevoir des redevances soit en espèces, soit mieux en nature, prélevables sur la récolte.

Si la marge entre le prix de vente moyen et le prix de revient minimum permet la perception de redevances suffisantes pour que toutes les charges soient couvertes, y compris les charges de premier établissement, tout sera parfait, ot il aura été réalisé une opération particulièrement brillante. Le cas est rare en France, à notre connaissance, sauf peut-être pour les rizières de Camargue, mais il se produit à l'étranger et les chiffres donnés par M. René Dumont sur les landes de Danemak montrent que l'Etat danois est largement rémunéré des dépenses qu'il a engagées.

Mais si une telle rentabilité, que nous pourrions appeler « rentabilité directe », n'est pas réalisée, cela ne veut pas dire que l'Etat, bailleur de fonds, ne fait pas une bonne opération financière. En effet, le développement d'une activilé nouvelle est la source de nombreux bénéfices nouveaux pour l'Etat, en particulier par l'intermédiaire du fisc. Il est vrai que cette activité nouvelle est également l'origine d'un certain nombre de dépenses supplémentaires. Dans chaque cas particulier important, où la rentabilitè directe n'est pas assurée, une élude de "rentabilité indirecte 》 devra être entreprise. Lorsque cela sera possible, elle aura pour base, d'une part, l'augmentation de production totale attendue et les résultats fiscaux obtenus dans des régions ayant subi des aménagements analogues, d'autre part, les augmentations de dépenses budgétaires dont on a constaté la nécessité dans d'autres régions déjà mises en valeur : frais d'administration, entretien des ouvrages publics, enseignement, santé, etc...

Lorsqu'on ne pourra pas trouver d'éléments statistiques relatifs à des aménagements passés, on aura quand même un élément en essayant de déterminer le revenu total des personnes qui vivent sur le périmètre et de celles dont lo chiffre d'affaires sera augmenté par suite de l'existence de ee périmètre et on appliquera ensuite la règle bien connue des économistes, d'après laquelle dans les pays modernes, le total des revenus de l'Etat est de l'ordre de grandeur du quart du revenu national; quant aux dépenses, on essayera de les analyser.

Suivant le résultat de ces diverses études 
économiques, le projet sera classé dans l'une des catégories suivantes :

Projet directement rentable : c'est celui où l'organisme chargé du périmètre étant supposé emprunter la somme peut rembourser l'emprunt à son prêteur, dans un délai raisonnable, et avec un taux d'intérêt normal.

Projet rentable indirectement : c'est celui où le prix de vente moyen à la production est supérieur au prix de revient minimum, mais où l'on doit faire intervenir les impôts de toute nature perçus par l'Etat pour assurer l'intérêt et l'amortissement des sommes investies.

Projet non rentable, mais productif : c'est celui où le prix de vente possible à la production est supérieur au prix de revient mais où il est impossible d'assurer, même indirectement, l'intérêt et l'amortissement des sommes investies.

Projet ni rentable, ni productif : c'est celui où le prix de vente possible est inférieur au prix de revient.

Il sera toujours sage d'éviter de réaliser des ouvrages correspondant au dernier cas, car il s'agirait alors de mesures d'assistance mettant certaines parties de la population à la charge de la nation toute entière.

Par contre, le choix entre divers projets productifs rentables ou non sera affaire de Gouvernement qui décidera en fonction de la productivité des investissements, de leur rentabilité budgétaire et des bénéfices d'ordre social qui en sont attendus.

Pour limiter au minimum les erreurs économiques, qui sont toujours très graves par leurs conséquences sociales, il est indispensable que dès le début des études techniques et pour toute la vie de l'exploitation, soit mis en place un dispositif d'auscultation économique. Ses statistiques porteront notamment sur les surfaces cultivées, les rendements de chaque campagne, les transactions caractéristiques du niveau de vie, le chiffre d'affaires des commerçants et industriels, les impôts, le nombre des bicyclettes et automobiles, la construction immobilière, etc... Enfin, l'évolution démographique devra être suivie avec attention.

\section{g) Eléments sociaux et pronlème foncier :}

Nous venons de dire que les résultats économiques des travaux ne sont pas seuls en cause mais qu'il faut tenir compte également de leurs conséquences sociales bénéfiques. L'élément humain intervient d'ailleurs de bien d'autres facons et il est indispensable que cet aspect de la question soit longuement étudié avant que soit prise une décision.
Tout d'abord, il faut préciser quelles considérations sociales interviennent dans la fixation des objectifs. Cela peut être la production de vivres destinés à des populations plus ou moins voisines et sous-alimentées; cela peut être aussi de donner du travail et des moyens de subsistance à des populations excédentaires; cela peut être enfin un objectif qui semble avoir un caractère purement économique tel que la production de denrées d'exportation mais qui, à un échelon plus élevé, permet en compensation l'importation de denrées de première nécessité à bon marché.

Mais l'élément social intervient également parmi les difficultés de mise en valeur, soit qu'on le considère sur le plan du travail, soit sur le plan de la propriété. En matière de maind'œuvre, la difficulté peut ètre une insuffisante densité de population ou des conditions maisaines de travail. On peut admettre qu'il est difficile même en culture mécanisée de descendre au-dessous d'un travailleur agricole pour trois hectares irrigués, ce qui pour les familles moyennes correspond à une densité de population de 100 habitants par kilomètre carré.

La difficulté peut être aussi un excédent de population sur le périmètre. A vrai dire, cette difficulté ne doit jamais être dirimante, car si elle entraine des complications résultant de la dimension des parcelles, on doit pouvoir y remédier par un régime foncier convenable et de toute façon puisque l'aménagement augmente la production, le niveau de vie d'une même quantité de personnes doit être amélioré.

Il peut arriver aussi que la population vivant sur le périmètre ne soit pas constituée par des agriculteurs susceptibles de s'adapter rapidement à des formes de cultures nouvelles. Là non plus, la question n'est pas très grave. Il faudra faire preuve de patience, admettre des délais qui peuvent être de plusieurs décades ou même d'une ou deux générations, mais il n'est pas vraisemblable que des populations attachées à la terre et ayant besoin de vivre, ne puissent pas s'adapter à des façons culturales nouvelles.

Enfin, une des grosses difficultés d'ordre social peut résulter de la répartition de la propriété du sol et du mode de tenure des terres. En fait, c'est là que réside la cause des trous d'une étude spéciale aux problèmes de tenure des terres: nous avons montré que la mise en valeur rationnelle suppose un certain nombre de servitudes, et avions proposé trois solutions suivant les objectifs de peuplement.

Il s'agira, bien entendu, dans chaque cas, d'une étude particulière, mais la puissance publique a le droit et le devoir de se montrer extrêmement ferme, tout en ayant le doigté nécessaire. Elle ne doit pas hésiter à prescrire les mesures conformes à l'intérêt général, étant 


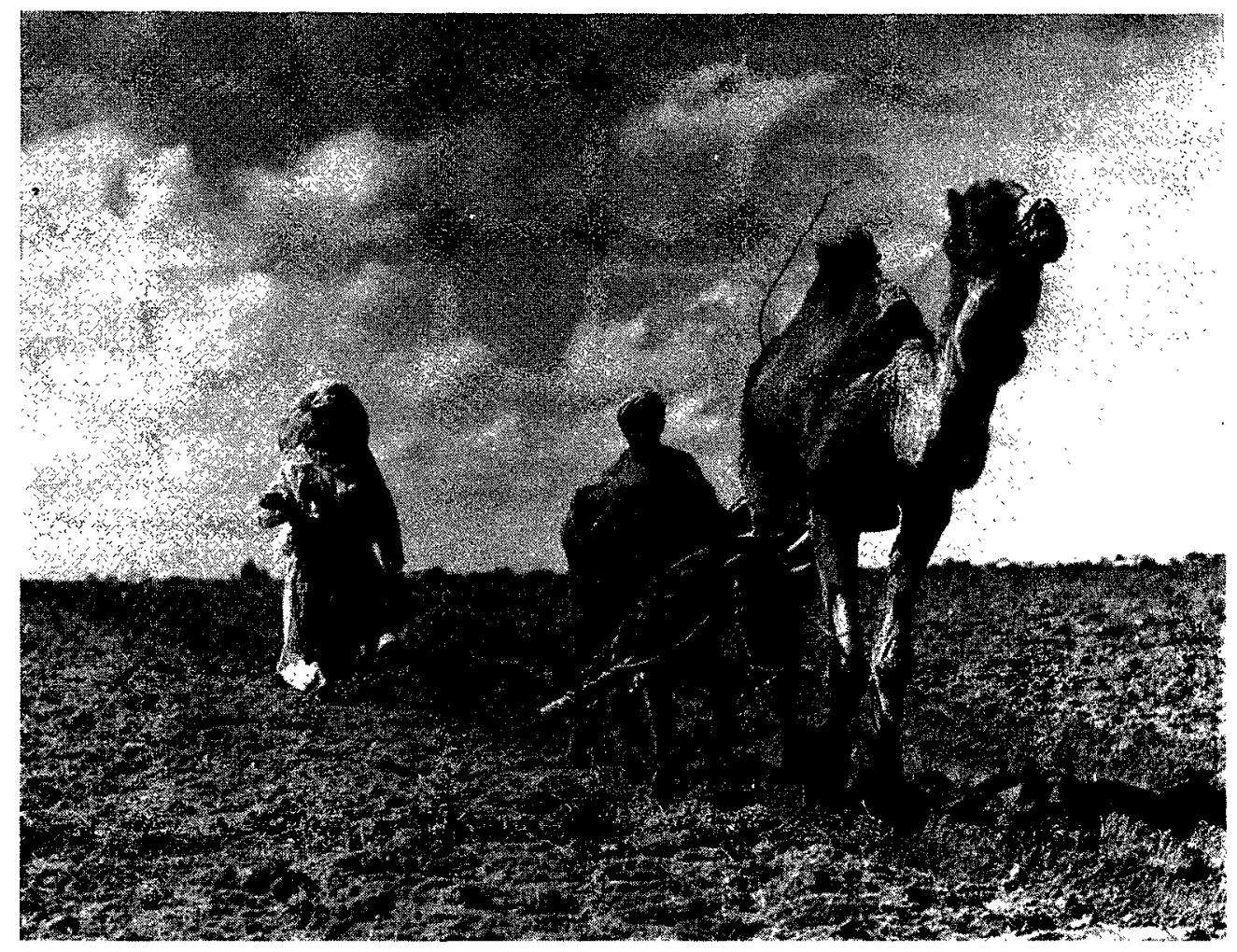

(Pholo J. Belin.)

Fig. 7. - Labour et semailles suivant la methode traditionnelle.

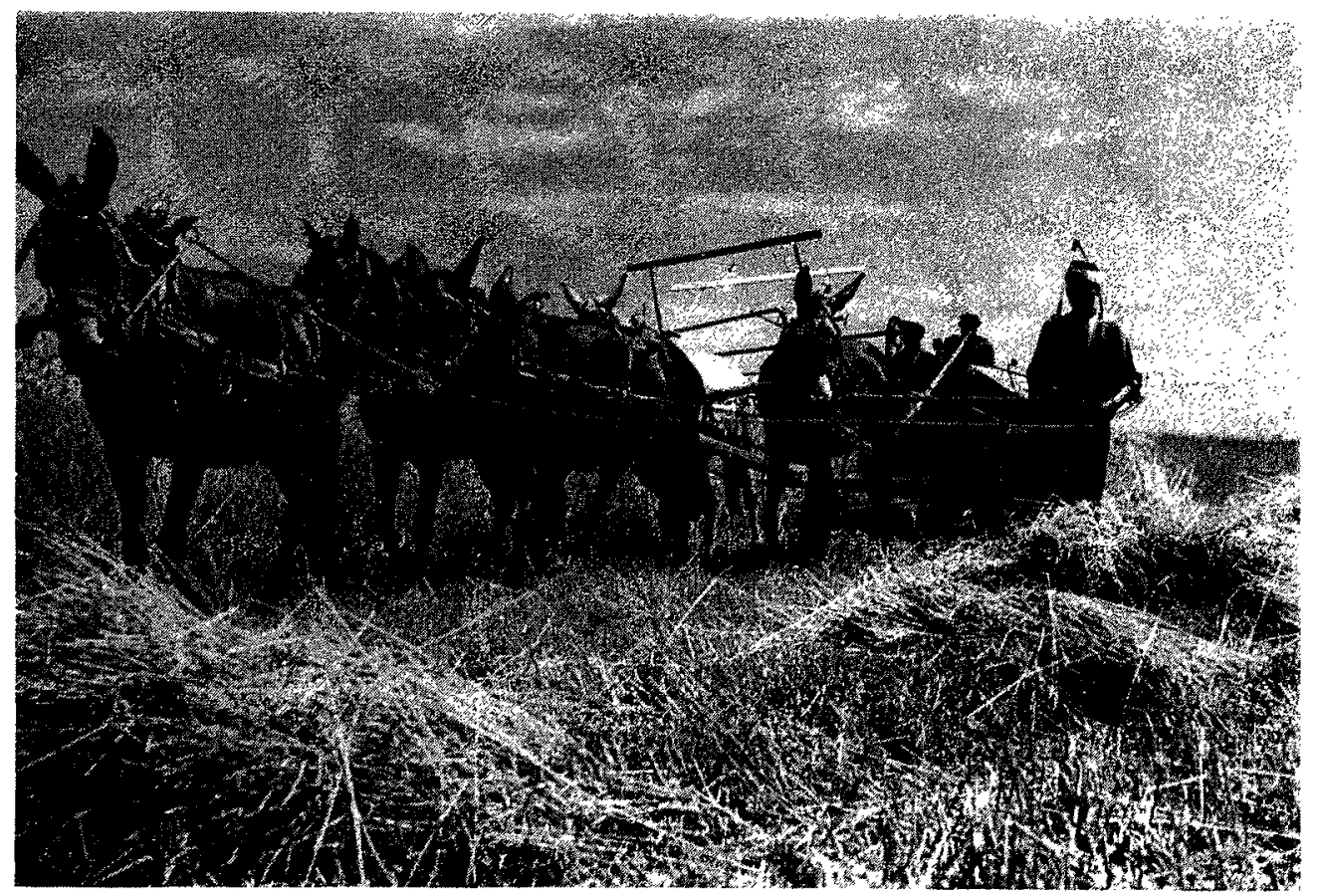

(Photo J. Belin.)

Fig. 8. -- Modernisation des méthodes de labour. 
bien entendu que les propriétaires privés d'un capital recevront un capital en numéraire et que les cultivateurs privés d'un moyen de subsistance recevront un moyen de subsistance équivalent.

\section{h) Divers stades des Études :}

La simple énonciation des différents éléments qu'il convient d'étudier pour dresser un projet cohérenl de mise en valeur par grands travaux d'hydranlique montre la complexité du problème, la multiplicité des organismes intéressés et fait prévoir que le coût de ces études sera élevé. En fait, lorsqu'il s'agit de travailler des dizaines de milliers d'hectares, le coût des études se chiffre par centaines de millions.

L'inter-action des différents services qui interviennent et le montant des études ainsi que les délais nécessaires à certaines d'entre elles, exigent le respect d'un certain nombre de stades dans la conduite de ces études.

Avant d'engager aucun frais important, même concernant les études, il faut dresser cc qu'on pourrait appeler le schéma chiflré. Mais même avant d'arrêter ce schéma, il y a lieu de réunir un certain nombre d'éléments d'informations qui, heureusement, ne coûtent pas cher, mais demandent du temps.

Ces études préliminaires devront comporter :

- des études hydrologiques portant sur plusieurs années,

- le plan de nivellement sommaire,

- l'étude climatologique,

-- l'étude pédologique,

- l'étude sociale,

- et une étude économique comprenant l'analyse du marché des produits susceptibles d'être récoltés.

L'intérêt de la navigation devra être évoqué, ainsi que les ressources et les débouchés pour l'électricité, le cas échéant;

En possession de ces éléments, il sera possible de dresser le programme chiffré comportant notamment :

-... l'évaluation du coût probable des divers ouvrages,

-- l'énumération des industries annexes et des réalisations sociales nécessaires,

-- et un projet de ventilation des dépenses entre l'électricité, la navigation et l'agriculture.

Ce programme devra permettre une conclusion provisoire concernant l'intérêt économique et social du projet et la mise en place éventuelle de centres pilotes ou stations agricoles, s'il sub- siste des inconnues importantes concernant l'agriculture.

C'est alors seulement que devront être accordés, en deuxième tranche, les crédits nécessaires à la préparation de l'avant-projet et la poursuite détaillée des études économiques, sociales ou agricoles qui s'avéreront nécessaires.

Cet avant-projet doit permeltre de connaître avec une grande précision (de l'ordre de $20 \%$ ) le coût des travaux, le coût probable de l'exploitation, la meilleure organisation pour l'exploitation $d u$ périmètre et les textes administratifs nécessaires.

C'est enfin lorsque l'avant-projet sera terminé et le cadre législatif de fonctionnement arrêté, que les crédits nécessaires à l'établissement du projet d'exécution pourront être accordés en même temps qu'on se préoccupera des crédits nécessaires à la réalisation du projet.

\section{i) Financement des études :}

D'après ce que nous venons de dire, il apparaît que les études préliminaires et le schéma peuvent être entrepris avant qu'on ait une idée précise de la rentabilité et des problèmes sociaux soulevés.

II n'est donc pas question de demander à un organisme privé ou para-administratif de payer de telles études, cela fait partie des devoirs de l'Etat de tenir à jour les possibilités de développement de la richesse nationale.

Il s'agit d'ailleurs de sommes faibles, de l'ordre de grandeur de quelques milliers de franes par hectare, mais de délais de plusieurs années.

Pour l'avant-projet encore les perspectives de succès sont trop incertaines et les projets d'organisation future trop imprécis pour qu'on puisse demander à un autre qu'à la puissance publique, de payer des dépenses qui restent modérées tout en étant plus élerées que les précédentes.

Les délais ici encore peuvent être longs, notamment si l'on envisage la mise en place de stations d'expérimentation.

Quant au projet d'exécution proprement dit, il ne doit, au contraire, être établi qu'après que le dispositif financier et légal aura été arrêté. II arrivera alors fréquemment que le coùt d'établissement de ce projet sera couvert par des méthodes analogues à celles employées pour les travaux proprement dits et que ce cont figurera parmi les dépenses de premier établissement.

\section{II. - Direction et coordination des études}

\section{(i) Organisme COORDinateur :}

La complexilé que nous avons montrée pour les études et le très grand champ qu'elles doivent 
embrasser nécessitent l'intervention de personnes physiques et morales de formation el d'origine très variées, mais il est impossible de faire un travail efficace sans qu'il existe un organisme chargé de diriger et de coordonner les diverses études spécialisées.

L'initiative d'un projet est souvent le fait d'un homme que sa profession ou sa situalion a familiarisé avec l'utilisation des caux (ingénieur des Ponts et Chaussées, ingénieur du Génie Rural, exploitant agricole, administrateur provenant des régions où l'on fait de l'irrigation).

La personne qui a eu l'idée peut la schématiser par quelques dessins et quelques ćvaluations. Si elle dispose de moyens techniques et financiers, elle peut même pousser assez loin l'évaluation du con̂t d'aménagement.

Quant aux résultats à en altendre, clle les appréciera fort mal, en général, sauf s'il s'agit d'un ingénieur agricole qui serait à la fois un économiste, mais dans ce cas c'est l'évaluation du coût des travaux qui se trouvera le plus souvent gravement erronée.

Dès ce stade. la collaboration apparaît donc nécessaire entre divers spécialistes, aussi le promoteur plein de la foi qui anime ordinairement le créateur, cherchera à convaincre soit les responsables des autres services intéressés, soit le gouvernement en la personne d'un de ses représentants : préfet, membre d'une grande organisation polyvalente ou même homme politique.

S'il réussit, il se constituera spontanément ou par décision administrative, un petit comité de deux ou trois techniciens, assistés ou non d'administrateurs ou d'utilisaleurs, et qui essaiera de dégager les divers problèmes qui peuvent se poser et la façon de les traiter. Nous n'en sommes pas encore aux études préliminaires et il est sage que jusqu'à ce stade aucun crédit d'études ne soit accordé.

C'est à la suite d'un rappork établi par l'organisme promoteur appuyé de notes ou de lettres des principaux autres services intéressés et d'une transmission du préfet que l'établissement d'un schéma chiffré pourra ĉtre décidé. La préparation de ce schéma sera suivie par un comité technique restreint, et soumis à la première des commissions d'examen dont il sera question plus loin.

Une fois le schéma retenu, le comité technique pourra être amené à s'adjoindre un petit nombre de membres, par exemple, un pédologue ou un hydrogéologue ou un industriel dans une branche correspondant aux productions prévues.

C'est ce nouveau comité, encore technique, qui dirigera et coordonnera les études de l'avantprojet.

Celui-ci, examiné par une deuxième commission d'examen et remanié, le cas échéant, servira de base au projet d'exécution.
A ce stade, le ròle moteur du comité technique est terminé. Il pourra être utile de lui conserver vie pour lui soumettre les projets d'execution, mais pendant toute la periode d'établissement de ces derniers, il y a tout intérêt à ce que les projets soient contrôlés par l'organisme qui dirigera les travaux.

\section{b) LIAISON AVEC LES SERVICES ET BUREAUX D'ÉTUDES ÉTRANGERS A L'ORGANiSME :}

Le comité technique, quoique comprenant dans son sein les principaux chefs de services intéressés, devra inviter à l'occasion de l'examen de telle ou telle question, les représeutants des bureaux d'études privés ou des divers services qui se seront chargés à titre gratuit ou à titre onéreux de telle ou telle partie des études.

Il est bon, par conséquent, que ce comilé dispose d'une certaine fraction de crédits d'études qui ne seront pas directement employes par les principaux services intéressés.

\section{III. - Commissions d'examen et jugement}

\section{a) Rìgles DIs COMMISSIONS croIss ANTES :}

S'il est indispensable que les comilis techniques ayant la charge de la direction et de la coordination des études ne comprennent pas d'éléments étrangers aux différentes spécialisatés en cause (et l'économie al la seience sociale se classent parmi les technicfues spécialisées), il est indispensable, au contraire, que les commissions chargées d'examiner les projets comprennent tout au moins à partir d'un certain stade, des utilisateurs qui peuvent laire connailre un avis, justifié ou non, et des hommes politiques qui sont à la fois les porte-parole de l'opinion publique et les soutiens des projets devant les asseinblées.

Celte règle des commissions croissantes est applicable en particulier dans le cas des grands travaux d'hydraulique agricole et il y a intérêt à ce que les différents stades des études soient examinés par différentes commissions dont la composition et le fonctionnement soul de plus en plus lourds.

Le schéma chiffré ne comporte pas d'éléments suffisamment certains pour qu'on puisse le soumettre à des non-lechniciens. I a premiere commission d'examen, celle chargée de retenir le schéma doit donc être composée uniquement de spécialistes des questions à étudier dans le domaine de l'Hydraulique, du Genje civil, de l'Agriculfure, de la Conservation des sols, de l'Administration et de la Sociologic. Ine commission de quatre ou cind membres, dont la composition serait analoguc à celle du comité 


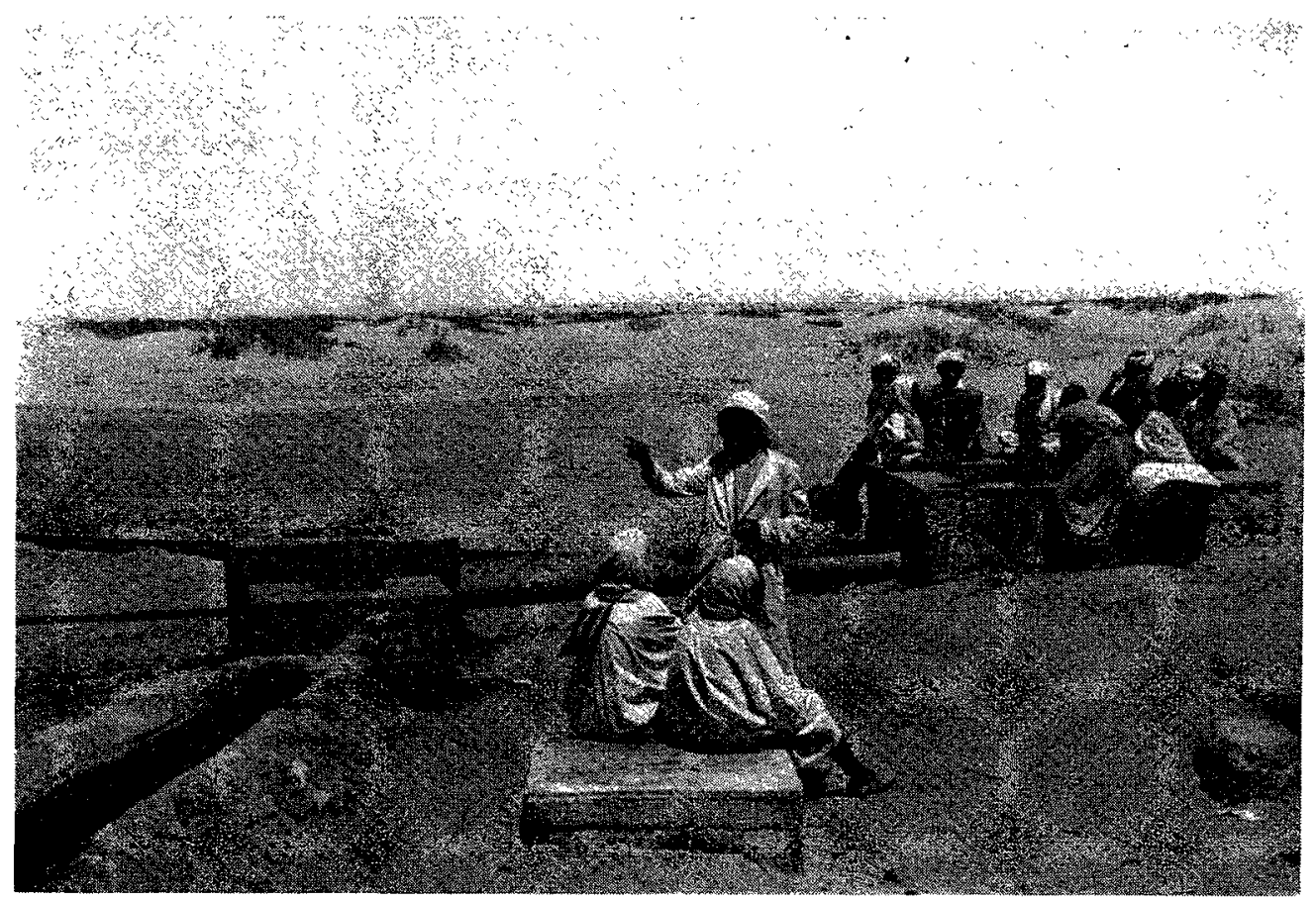

Fig. 9. - Région de Ksar Rhilane avant irrigation (travaux en cours).

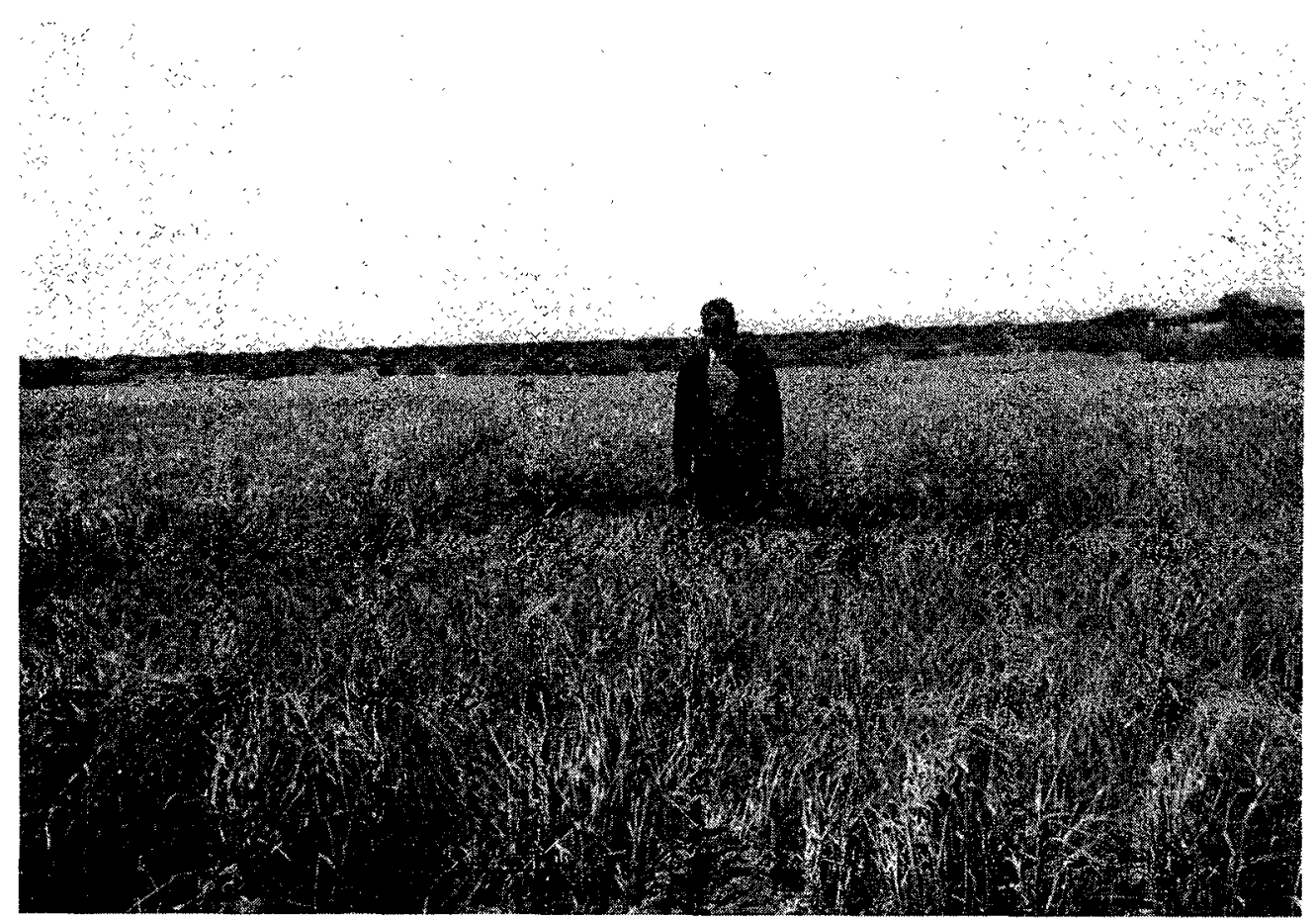

F1G. 10. - Région de Ǩsar Rhilane après irrigation. 
d'étude, mais constituée, à Paris, par de hautes personnalités des techniques intéressées, semble convenir parfaitement.

Les membres du comité technique assisteraient de droit aux réunions de la commission sans avoir voix délibérative.

L'avant-projet, dont les conclusions doivent être solidement étayées, serait cxaminé par une commission plus étoffée comprenant notamment des représentants de l'administration des Finances, des conseillers généraux, des représentants des professions intéressées et des nembres des grandes assemblées.

\section{b) DÉcISION :}

Ces diverses commissions ne peuvent pas avoir pouvoir de décision puisqu'il s'agit de deniers de l'Etat dont elles ne disposent pas.

La décision résultera d'un acte du pouvoir central qui sera pris par un ministre, par un arrêté interministériel, par un décret ou par une loi, suivant le nombre de ministères intéressés $\mathrm{cl}$ l'importance des questions sociales ou domaniales mises en cause.
Quant au projet d'exécution proprement dit, il portera généralement sur des points précis et très nombreux qui ne peuvent pas intéresser les hautes instances une fois l'avant - projet approuvé. Il appartiendra donc aux représenlants qualifiés des ministères intéressés d'approuver chacune des parties du projet d'exécuiion, tant sur le plan technique que sur le plan administralif. Lorsqu'un point intéressera deux services différents, l'approbation devra être conjointe.

\section{Précautrons pour éviter la spéculation}

Il est arrivé souvent que des spéculateurs bien informés exploitent l'ignorance des paysans pour leur acheter les terres dont l'irrigation est envisagée. Le prix qu'ils offrent est supérieur à la valeur de la terre sèche, mais hien inférieur à celle de la terre irrigable.

Il sera sage de prendre des mesures conservatoires interdisant tout achat dès la décision d'approbation du schéma chifré et pour l'acte mème qui approuve ce schéma.

(A suivre.)

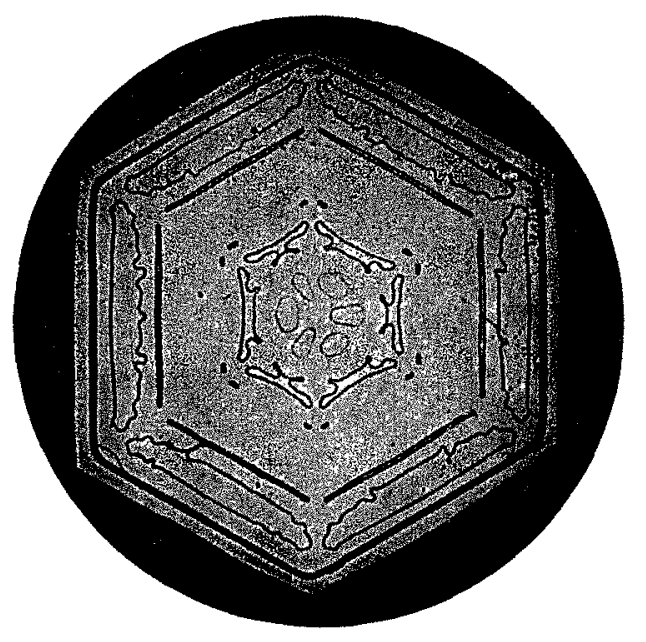

